\title{
Asthma: 1987-2007. What have we achieved and what are the persisting challenges?
}

\author{
*Martyn R Partridge \\ Professor of Respiratory Medicine, Imperial College London, NHLI Division, Charing Cross Hospital, London, UK
}

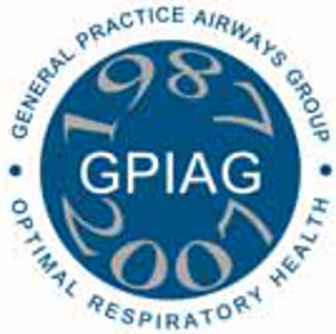

Received 25th April 2007; accepted 8th May 2007

\section{Summary}

Despite an increasing prevalence of asthma, enormous advances have been made in our understanding and management of asthma over the last 20 years. Work begun two or three decades ago demonstrated the inflammatory nature of asthma, emphasised the need for regular treatment, and introduced the goal of maintaining normal lung function. More recent work demonstrated the benefits of adding a long-acting inhaled beta-agonist to low-dose inhaled steroids as an alternative to escalating the steroid dosage. More recently still, studies and regulatory approval have led to the possibility of the same inhaler being used for maintenance therapy and for relief. However, these new ways of using old medicines, along with some new medicines such as omalizumab, should not detract us from looking beyond the prescription. The challenges facing us now are to determine why the prevalence of asthma has increased so dramatically, and in the absence of a cure how we can best help increasing numbers of people with asthma benefit from the treatment which is available. This will involve a much more aggressive implementation of advice regarding self-management education and a restructuring of health services to provide a service that is cognisant of the fact that, like us, patients are increasingly busy - and if they are to benefit from regular review, that review needs to be offered at a convenient time and by convenient methods.

(C) 2007 General Practice Airways Group. All rights reserved.

MR Partridge. Prim Care Resp J 2007; 16(3): 145-148.

doi:10.3132/pcrj.2007.00039

Keywords asthma, management, treatment, guidelines, challenges

\section{Contents}

Introduction

The mid-1980s

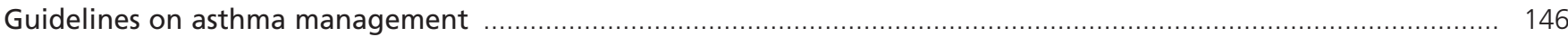

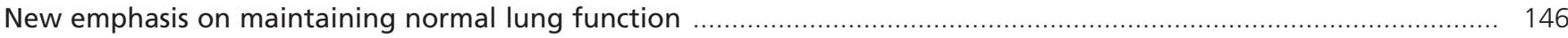

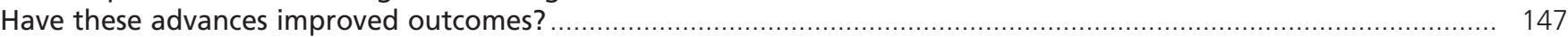

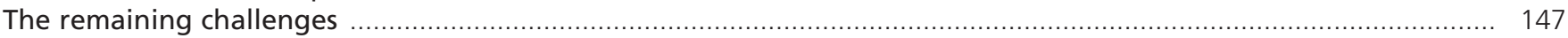

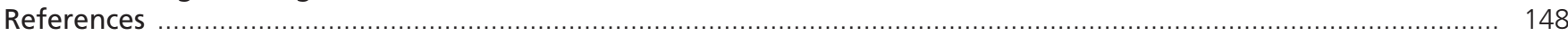

\section{Introduction}

The burden of respiratory disease has moved imperceptibly over the last few decades from a burden of respiratory infections to a burden of long-term conditions such as chronic obstructive pulmonary disease (COPD), sleep apnoea syndromes, diffuse parenchymal lung disease and asthma. The latter affects an increasing proportion of the population globally and as yet we do not have the knowledge to enable us to develop a meaningful primary prevention strategy. Management of the condition was dramatically improved when treatments such as adrenaline and oral steroids were joined by inhaled steroid therapy, but three decades ago there was little publicity about the condition, negligible information materials available for patients, little in the way of structured care, and no guidelines on correct management for health professionals. The last two decades have therefore been times of change, but many challenges remain.

\section{The mid-1980s}

Twenty years ago, many parts of the world were experiencing the second peak of asthma deaths of the millennium. Hospital

* Corresponding author: Charing Cross Hospital, St Dunstan's Road, London W6 8RP, UK

Tel: +44 (0)20 88467181 Fax: +44 (0)20 88467999 Email: m.partridge@imperial.ac.uk 
admissions for asthma were rising rapidly, especially in the young, and serial studies were beginning to show a global epidemic of asthma. 'We had no guidelines to sift for us the evidence in favour of different management approaches, but the studies which had been undertaken in the 1970s on the value of inhaled beclomethasone had been followed by studies suggesting that for those who were not well controlled there were benefits in using a higher dose of beclomethasone. ${ }^{2}$

However, the fact that the available treatments were not suitable for all had led to trials of alternatives, with drugs such as methotrexate being shown to lead to a significant reduction in the use of oral steroids in those at the severe end of the asthma spectrum. ${ }^{3}$ Other aspects of difficult asthma - such as premenstrual exacerbations - were also being highlighted, with reports of the benefits to be gained from intra-muscular progesterone. ${ }^{4}$

New inhaler devices to deliver therapy directly to the airways were also being evaluated; a 1989 Drug and Therapeutics Bulletin concluded that conventional metered dose inhalers (MDIs) were to remain the first line treatment, but also commented (with some foresight) that "breath-actuated powder systems may eventually supersede metered dose inhalers because they avoid the use of chloro-fluoro carbon propellants". ${ }^{5}$ The risk versus the benefits of therapies were also being aired, with case reports of cataract formation in those who were treated with steroid aerosol inhalers - although it was difficult to disentangle this effect from concomitant use of steroid tablets. ${ }^{6}$ A more worrying risk-versusbenefit argument was to arise at the end of the decade with case control studies suggesting that the use of fenoterol by MDI increased the risk of death in severe asthma.?

\section{Guidelines on asthma management}

In mid-1989, Brian Harrison and I, with the support of the British Thoracic Society, Asthma UK, and the late Anthony Hopkins, director of the research unit of the Royal College of Physicians in London, began the process of producing the first British Guidelines for the good management of asthma in adults, which were published in the British Medical Journal. ${ }^{8}$ These guidelines, which were produced around the same time as similar statements in Australia and Canada, promoted a stepwise approach to the management of asthma, such that if a bronchodilator was required more than occasionally the patient should start inhaled anti-inflammatory agents - which would normally be inhaled steroids - and that these would be increased if control was not achieved. At Step 4 additional bronchodilators would be added and the concept of stepping down treatment was recommended, as was the suggestion that "as far as possible, patients should be trained to manage their own treatment rather than be required to consult their doctor before making changes." Self-management plans were similarly recommended and what they should contain was clearly spelt out.

\section{Table 1. The achievements of the last decade.}

- Understanding the inflammatory nature of the disease

- Understanding the value of trials of additional longacting inhaled beta-agonists before increasing the dose of inhaled steroids

- Appreciating the value of self-management education (but not always implementing it)

- Learning how to use existing medications in a manner which is advantageous to our patients (new devices, combination inhalers, single inhaler maintenance and reliever regimens)

\section{New emphasis on maintaining normal lung function}

The early 1990s saw a series of studies involving the pathogenesis of disease, its natural history, and new pharmacological approaches, which still underpin our management approach today. Work from Finland published just over 20 years ago began to demonstrate that structural changes and inflammation were present in the airways of our patients with asthma, even at a time when they were relatively well. ". The same scientists subsequently demonstrated how those who were treated more promptly with inhaled steroids did better than those who were initially treated with bronchodilators alone and showed that the latter group did not recover normal function even when they were then switched to inhaled steroids. ${ }^{10}$

Whilst these studies encouraged a lower threshold for the initiation of treatment with inhaled steroids, a series of studies then began to question the wisdom of the progressive increase in the inhaled steroid dose in those patients with sub-optimally controlled asthma, suggesting that the addition of salmeterol may produce better results. ${ }^{11,12}$ When these studies were followed by similar ones showing that the same applied when formoterol was added to a low dose of various other inhaled steroids, the scene was set for guidelines to recommend that before increasing the dose of inhaled steroids one should recommend a trial of a longacting inhaled beta-agonist.

For the majority of those with asthma the therapeutic recommendations which we currently adopt are thus based upon studies undertaken in the decade 1986 - 1995. The achievements in asthma management over the last decade are outlined in Table 1. Subsequent studies on leukotriene modifiers, the benefits of additional theophyllines, and new modalities of treatment such as omalizumab, have represented introductions which are either of limited benefit or applicable to only small numbers of patients. The current position with regards to treatment is well laid out in national and international guidelines with the British Guideline being subjected to a rigorous evaluative process which is dynamic and associated with regular revisions. ${ }^{13}$ 


\section{Table 2. Challenges for the next two decades.}

- Understanding why asthma has increased in prevalence and devising effective primary prevention strategies

- Simplifying diagnosis but making sure that a firm diagnosis is made wherever possible before initiating therapy, or otherwise recognising that such therapy is a diagnostic trial of therapy

- Ensuring that those with 'difficult' asthma are properly and fully evaluated

- Fully implementing recommendations regarding selfmanagement education

- Recognising that asthma is a long-term condition and that patients are as busy as we are, and offering easier methods of review at convenient times.

\section{Have these advances improved outcomes?}

So have these advances reduced the burden of asthma, and what are the current challenges? Using traditional parameters of outcomes, there are some grounds for optimism in that despite the epidemic of asthma and the increased prevalence of asthma in many countries, the death rate has fallen and the hospitalisation rate for children at least has fallen.

However, significant hospital admissions still occur at all ages and - especially amongst adults - there has been little recent change. Indeed, in some countries there is worrying evidence that the numbers needing unscheduled health care from emergency departments has not changed at all over the last few years. Furthermore, morbidity studies suggest that despite guidelines laying out clear optimal outcomes for the management of asthma, these are not met in many countries. ${ }^{14}$ In some of these studies only a quarter of patients were on maintenance therapy and it wasn't clear whether the ongoing morbidity was due to the inadequacy of treatment or the failure of it to be prescribed. More recent studies have suggested that even in those who are on regular maintenance therapy there is persisting morbidity which is often underestimated by patients. ${ }^{15}$ This emphasises the importance of always asking specific questions designed to elucidate poor asthma control when patients attend for consultations. Whether or not guidelinedefined asthma control can be achieved has indeed been studied, and better results are clearly obtainable in a majority of patients, although there are a significant number for whom current therapies do not resolve the problem. ${ }^{16}$

Newer modalities of treatment are being assessed. Omalizumab, a humanised monoclonal antibody given by injection at two-weekly intervals, is a possible add-on therapy in adults and adolescents with severe persistent allergic asthma and appropriate total IgE levels. ${ }^{17}$ Whether non-pharmacological interventions such as the application of thermal energy through a bronchoscope will figure prominently in future management remains to be seen..$^{18}$ More likely is the situation that different ways of using existing therapies will prove to be better tolerated and more easily taken by patients. Such an approach has recently been given regulatory approval in the EU and elsewhere, whereby a budesonide/ formoterol combination inhaler is used regularly as maintenance therapy but also on an as-needed basis for relief of symptoms. This approach is likely to be attractive to patients because of its ease of use, and most of the studies have suggested that such an approach is associated with a reduction in exacerbation rate and less need for steroid tablets. ${ }^{19}$

\section{The remaining challenges}

The challenges for the next two decades are outlined in Table 2 . They include offering existing therapies to patients in a manner which makes it likely that the treatments are used to maximal effect - but the challenges are wider than those of the prescription. Prompter, more accurate diagnosis of asthma is one challenge. We do not have a simple diagnostic test such as a level of blood sugar or blood pressure which defines disease, and there is likely to be continuing over- and under-diagnosis of asthma and delays before appropriate therapy is initiated. A diagnostic definition such as "demonstration of generalised airway narrowing, which varies over short periods of time, either spontaneously or as a result of treatment" - as outlined in the original CIBA symposium - is a good starting point, and may remain more practical and realistic than newer techniques looking at inflammatory markers, sputum eosinophils or demonstration of airway hyper-responsiveness.

A second challenge is to ensure that accurate diagnosis and accurate evaluation of severity and co-morbidity is made in those patients at the severe end of the spectrum. Approaches to those with difficult asthma have been shown to be different between specialists with an expressed special interest in difficult asthma compared to that adopted by other respiratory physicians, and a structured protocol-based approach is often necessary if mistakes in diagnosis and the overlooking of co-morbidity is to be avoided. ${ }^{20}$

We also need to recognise that our patients will live with their asthma for decades, and it is they who must become the experts in the management of their disease; yet all too often we fail to offer them the simple tools, skills and knowledge which they need to manage their condition, even when they want to do so. ${ }^{21}$ If doctors and nurses still fail to address this important issue and fail to implement guideline recommendations on self-management, other direct approaches to empower patients will have to be made. But even those who feel confident to self-manage their own asthma need our advice, support and review from time to time. A further challenge is to recognise that the services which we can offer are rarely truly patient-centred, and more consideration needs to be given to out-of-hours clinics and telephone review. ${ }^{22,23}$ 
However, none of these challenges would remain relevant if we had available a cure, or if we had a method of preventing the onset of this disease. These are the ultimate challenges for the next two decades.

\section{Conflict of interest declaration}

The author has received fees for lecturing, ad hoc consultancy, support for research, and sponsorship to attend international meetings, from AstraZeneca, Novartis, GSK, Cipla, Chiesi and Medapharma.

\section{References}

1. Devenny A, Wassall H, Ninan T, Omran M, Khan SD, Russell G. Respiratory symptoms and atopy in children in Aberdeen: questionnaire studies of a defined school population repeated over 35 years. BMJ 2004;329(7464):489-90.

2. Smith MJ, Hodson ME. High-dose beclomethasone inhaler in the treatment of asthma. Lancet 1983;5(1):265-9.

3. Mullarkey MF, Blumenstein BA, Andrade WP, Bailey GA, Olason I, Wetzel CE. Methotrexate in the treatment of corticosteroid-dependent asthma. A doubleblind crossover study. N Engl J Med 1988;318(10):603-07.

4. Beynon HL, Garbett ND, Barnes PJ. Severe premenstrual exacerbations of asthma: effect of intramuscular progesterone. Lancet 1988;2(8607):370-2.

5. Delivery systems for inhaled drugs in asthma. Drug Ther Bull 1989;27(17):65-8.

6. Allen MB, Ray SG, Leitch AG, Dhillon B, Cullen B. Steroid aerosols and cataract formation. BMJ 1989;299(6696):432-3.

7. Crane J, Pearce N, Flatt A, et al. Prescribed fenoterol and death from asthma in New Zealand, 1981-83: case-control study. Lancet 1989;1(8644):917-22.

8. British Thoracic Society, Research Unit of the Royal College of Physicians of London, Kings' Fund Centre, National Asthma Campaign. Guidelines for management of asthma in adults: 1 - Chronic persistent asthma. BMJ 1990; 301:651-3.

9. Laitinen LA, Heino M, Laitinen A, Kava T, Haahtela T. Damage of the airway epithelium and bronchial reactivity in patients with asthma. Am Rev Respir Dis 1985; 131(4):599-606.

10. Haahtela $T$, Jarvinen $M$, Kava $T$, et al. Comparison of a beta 2-agonist, terbutaline, with an inhaled corticosteroid, budesonide, in newly detected asthma. N Engl J Med 1991;325(6):388-92.
11. Greening AP, Ind PW, Northfield M, Shaw G. Added salmeterol versus higherdose corticosteroid in asthma patients with symptoms on existing inhaled corticosteroid. Allen \& Hanburys Limited UK Study Group. Lancet 1994; 344(8917):219-24.

12. Woolcock A, Lundback B, Ringdal N, Jacques LA. Comparison of addition of salmeterol to inhaled steroids with doubling of the dose of inhaled steroids. Am J Respir Crit Care Med 1996;153(5):1481-8.

13. British Thoracic Society, Scottish Intercollegiate Guidelines Network. British Guideline on the Management of Asthma. Thorax 2003;58:i1-i94.

14. Rabe KF, Vermeire PA, Soriano JB, Maier WC. Clinical management of asthma in 1999: the Asthma Insights and Reality in Europe (AIRE) study. Eur Respir $J$ 2000; 16(5):802-07.

15. Partridge MR, van der MT, Myrseth SE, Busse WW. Attitudes and actions of asthma patients on regular maintenance therapy: the INSPIRE study. BMC Pulm Med 2006;6:13.

16. Bateman ED, Boushey HA, Bousquet J, et al. Can guideline-defined asthma control be achieved? The Gaining Optimal Asthma ControL study. Am J Respir Crit Care Med 2004;170(8):836-44.

17. Holgate ST, Chuchalin AG, Hebert J, et al. Efficacy and safety of a recombinant anti-immunoglobulin E antibody (omalizumab) in severe allergic asthma. Clin Exp Allergy 2004;34(4):632-8.

18. Brown RH, Wizeman W, Danek C, Mitzner W. Effect of bronchial thermoplasty on airway distensibility. Eur Respir J 2005;26(2):277-82.

19. O'Byrne PM, Bisgaard H, Godard PP, et al. Budesonide/formoterol combination therapy as both maintenance and reliever medication in asthma. Am J Respir Crit Care Med 2005;171(2):129-36.

20. Roberts NJ, Robinson DS, Partridge MR. How is difficult asthma managed? Eur Respir J 2006;28(5):968-73.

21. Haughney J, Barnes G, Partridge M, Cleland J. The Living and Breathing Study: A study of patients' views of asthma and its treatment. Prim Care Resp J 2004; 13(1):28-35. doi:10.1016/j.pcrj.2003.11.007

22. Feeney CL, Roberts NJ, Partridge MR. Do medical outpatients want 'out of hours' clinics? BMC Health Serv Res 2005;5(1):47.

23. Pinnock $H$, Bawden $R$, Proctor $S$, et al. Accessibility, acceptability, and effectiveness in primary care of routine telephone review of asthma: pragmatic, randomised controlled trial. BMJ 2003;326:477-9.

Available online at http://www.thepcrj.org 